\title{
Karakteristik Subsemiring Fuzzy
}

\author{
Saman Abdurrahman \\ Program Studi Matematika FMIPA Universitas Lambung Mangkurat, JI. A. Yani Km 36 Banjarbaru \\ Kalimantan Selatan 70714, Indonesia.
}

Korespondensi; Saman Abdurrahman, Email: saman@ulm.ac.id

\begin{abstract}
Abstrak
Dalam tulisan ini, didefinisikan subsemiring fuzzy dan menyelidiki sifat yang terkait. Selain itu, diperkenalkan konsep subsemiring yang diinduksi dari level subset. Akhirnya, karakteristik subsemiring fuzzy diperoleh.
\end{abstract}

Kata Kunci: Semiring; subsemiring; subset fuzzy; level subset, subsemiring fuzzy.

\begin{abstract}
In this paper, we define the notion of fuzzy subsemiring and investigate the related properties. Moreover, we introduce the idea of a subsemiring induced from the level subset. Finally, a characterization of a fuzzy subsemiring is obtained.
\end{abstract}

Keywords: Semiring; subsemiring; fuzzy subset; level subset; fuzzy subsemiring.

\section{Pendahuluan}

Konsep subgrup fuzzy pertama kali diperkenalkan oleh Rosenfeld [1]. Konsep ini, merupakan perpaduan antara konsep teori grup dan himpunan fuzzy yang diperkenalkan oleh Zadeh [2]. Konsep subgrup fuzzy, merupakan konsep dasar dari aljabar fuzzy yang dijadikan oleh peneliti selanjutnya sebagai pondasi dalam membangun dan menyelidiki sifat dari struktur aljabar fuzzy lainnya, diantaranya: interior semiring fuzzy diperkenalkan oleh Mandal [3]; dan konsep interior subgrup fuzzy yang dikaitkan dengan homomorfisma grup diperkenalkan oleh Abdurrahman [4]; dan konsep ideal fuzzy pada gamma near-ring diperkenalkan oleh Jun at al [5].

Pada tulisan ini, akan menyelidiki sifat-sifat dasar dari subsemiring fuzzy yang dibangun oleh level subset ataupun subset dari semiring yang dikaitan dengan nilai keanggotaan elemen nol pada operasi pertama. Sifat-sifat subsemiring fuzzy yang diselidiki, diinduksi dari penelitian Abdurrahman [6] dan Jun at al [5].

\section{Landasan Teori}

Semiring merupakan salah satu perluasan dari ring. Menurut Ahsan at al [7], suatu himpunan $S(\neq \varnothing)$ dikatakan membentuk semiring, jika pada $S$ didefinisikan dua operasi biner penjumlahan $(+)$ dan perkalian $(\cdot)$ sedemikian sehingga $(S,+)$ adalah semigrup abelian, dan $(S, \cdot)$ adalah semigrup (tidak harus komutatif), serta dipenuhi sifat distributif kiri dan kanan, yaitu:

$$
b(a+c)=b a+b c, \text { dan }(b+c) a=b a+c a .
$$

untuk setiap $a, b, c \in S$. Jika semigrup $(S, \cdot)$ memuat elemen identitas 0 , maka elemen identitas ini disebut elemen nol pada semiring $(S,+, \cdot)$ sedemikian sehingga untuk setiap $c \in S$ berlaku:

$$
c+0=0+c=c \text { dan } c \cdot 0=0 \cdot c=0 .
$$


Seperti pada ring terdapat subring, pada semiring juga terdapat subsemiring seperti yang didefinisikan oleh Jagatap [8]. Subset $K(\neq \emptyset)$ dari semiring $S$ disebut subsemiring dari $S$, jika

untuk setiap $b, c \in S$.

$$
b+c \in K \text { dan } b c \in K
$$

Definisi 2.1 [9] Subset fuzzy $\rho$ dari himpunan tidak kosong $S$ adalah fungsi dari $S$ ke interval tutup $[0,1]$.

Definisi 2.2 [9] Misalkan $\rho$ adalah subset fuzzy dari $S$ dan $a \in[0,1]$ sedemikian sehingga

Himpunan $\rho_{a}$ disebut level subset dari $\rho$.

$$
\rho_{a} \stackrel{\text { def }}{=}\{x \in S \mid \rho(x) \geq a\} \text {. }
$$

Definisi 2.3 [7] Subset fuzzy $\rho$ dari semiring $S$ disebut subsemiring fuzzy dari $S$ jika untuk setiap $a, b \in S$ berlaku

$$
\rho(a+b) \geq \rho(a) \wedge \rho(b) \text { dan } \rho(a b) \geq \rho(a) \wedge \rho(b) .
$$

\section{Hasil dan Pembahasan}

Teorema 3.1 Misalkan $S$ adalah semiring yang memuat elemen $O$ dan $\rho$ adalah subsemiring fuzzy dari semiring $S$. Jika $\rho(0) \geq \rho(w)$ untuk setiap $w \in S$, maka himpunan

adalah subsemiring dari $S$.

$$
S_{\rho} \stackrel{\text { def }}{=}\{z \in S \mid \rho(z)=\rho(0)\}
$$

\section{Bukti:}

Misalkan $S$ adalah semiring yang memuat elemen 0 dan $\rho$ adalah subsemiring fuzzy dari semiring $S$, maka berdasarkan definisi $S_{\rho}$, diperoleh $S_{\rho} \subseteq S$ dan $\rho(0)=\rho(0)$, sehingga $0 \in S_{\rho}$, yaitu $S_{\rho} \neq \emptyset$. Selanjutnya, diambil sembarang $x, w \in S_{\rho}$, maka $\rho(x)=\rho(0)$ dan $\rho(w)=\rho(0)$. Akibatnya, berdasarkan Definisi 2.3 diperoleh

Oleh karena itu,

$$
\rho(x+w) \geq \rho(x) \wedge \rho(w)=\rho(0) \text { dan } \rho(a b) \geq \rho(a) \wedge \rho(b)=\rho(0) .
$$

Dengan kata lain, $S_{\rho}$ adalah subsemiring dari $\mathrm{S}$.

$$
x+w \in S_{\rho} \text { dan } x w \in S_{\rho} .
$$

Teorema 3.2 Misalkan $R$ adalah subset tidak kosong dari semiring $S$ dan $\rho_{R}$ subset fuzzy dari $S$ yang didefinisikan dengan

$$
\rho_{R}(x)= \begin{cases}a, & x \in R \\ b, & x \in S \backslash R\end{cases}
$$

untuk setiap $x \in S$ dan $a, c \in[0,1]$ dengan $a>b$, maka $\rho_{R}$ adalah subsemiring fuzzy dari $S$ jika dan hanya jika $R$ adalah subsemiring dari $S$.

\section{Bukti:}

$\left(\Rightarrow\right.$ ) Diambil sembarang $x, w \in R$, maka $\rho_{R}(x)=a$ dan $\rho_{R}(w)=a$. Karena $\rho_{R}$ adalah subsemiring fuzzy dari $S$ dan $R \subseteq S$, diperoleh

$$
\rho_{R}(x+w) \geq \rho_{R}(x) \wedge \rho_{R}(w)=a \text { dan } \rho_{R}(x w) \geq \rho_{R}(x) \wedge \rho_{R}(w)=a .
$$

Oleh karena itu, $x+w \in R$ dan $x w \in R$. Dengan kata lain $R$ adalah subsemiring dari $S$.

$(\Leftarrow)$ Misalkan $\mathrm{R}$ adalah subsemiring dari $\mathrm{S}$ dan $\rho_{R}$ subset fuzzy dari $S$. Diambil sembarang $x, w \in S$, maka dapat ditinjau untuk beberapa kasus, yaitu:

1) $x, w \in S \backslash R$, maka $\rho_{R}(x)=b$ dan $\rho_{R}(w)=b$, yang mengakibatkan

$$
\rho_{R}(x+w) \geq b=\rho_{R}(x) \wedge \rho_{R}(w) \text { dan } \rho_{R}(x w) \geq b=\rho_{R}(x) \wedge \rho_{R}(w) .
$$


2) $x \in R$ dan $w \in S \backslash R$, maka $\rho(x)=a$ dan $\rho(w)=b$, yang mengakibatkan

$$
\rho_{R}(x+w) \geq b=\rho_{R}(x) \wedge \rho_{R}(w) \text { dan } \rho_{R}(x w) \geq b=\rho_{R}(x) \wedge \rho_{R}(w) .
$$

3) $x, w \in R$, maka $x+w \in R$ dan $x w \in R$, sehingga $\rho_{R}(x)=a, \rho_{R}(w)=a, \rho_{R}(x+w)=a$ dan $\rho_{R}(x w)=a$, yang mengakibatkan

$$
\rho_{R}(x+w)=a=\rho_{R}(x) \wedge \rho_{R}(w) \text { dan } \rho_{R}(x w)=a=\rho_{R}(x) \wedge \rho_{R}(w) .
$$

Berdasarkan hasil analisa di atas, untuk setiap $x, w \in S$ berlaku

$$
\rho_{R}(x+w) \geq \rho(x) \wedge \rho(w) \text { dan } \rho_{R}(x w) \geq \rho(x) \wedge \rho(w) .
$$

Dengan kata lain, $\rho_{R}$ adalah subsemiring fuzzy dari $S$.

Akibat 3.3 Misalkan $R(\neq \emptyset)$ adalah subset dari semiring $R$ dan $\chi_{R}$ adalah fungsi karakteristik dari $R$, maka $\chi_{R}$ adalah subsemiring fuzzy dari $S$ jika dan hanya jika $R$ adalah subsemiring dari $S$.

Teorema 3.4 Subset fuzzy $\rho$ dari semiring $S$ adalah subsemiring fuzzy dari $S$ jika dan hanya level subset $\rho_{a}(\neq \emptyset)$ adalah subsemiring dari $S$, untuk setiap $a \in \rho(S)$.

\section{Bukti:}

( $\Rightarrow$ ) Misalkan $\rho$ adalah subsemiring fuzzy dari semiring S. Akan dibuktikan subset tidak kosong $\rho_{a}$ dari $\mathrm{S}$ adalah subsemiring dari $\mathrm{S}$, untuk setiap $a \in \rho(S)$. Diambil sembarang $x, z \in \rho_{a}$, maka $\rho(x) \geq$ $a$ dan $\rho(z) \geq a$. Karena $\rho$ adalah subsemiring fuzzy dari $\mathrm{S}$ dan $\rho_{a}$ subset dari $\mathrm{S}$, maka $x, z \in S$, sehingga berdasarkan Definisi 6, diperoleh kondisi berikut ini.

Oleh karena itu,

$$
\rho(x+z) \geq \rho(x) \wedge \rho(z) \geq a \text { dan } \rho(x z) \geq \rho(x) \wedge \rho(z) \geq a .
$$

$$
x+z \in \rho_{a} \text { dan } x z \in \rho_{a} .
$$

Berdasarkan hasil Analisa di atas, $\rho_{a}$ adalah subsemiring dari $\mathrm{S}$ untuk setiap $a \in \rho(S)$.

$(\Leftarrow)$ Misalkan $\rho_{a}$ adalah subsemiring dari $S$, untuk setiap $a \in \rho(S)$ dan $\rho$ subset fuzzy dari $S$. Akan dibuktikan $\rho$ adalah subsemiring fuzzy dari $S$. Diambil sembarang $z, w \in S$, maka ada $a, c \in \rho(S)$ sedemikian sehingga $\rho(z)=a$ dan $\rho(w)=c$. Misalkan $d=a \wedge c$, maka $\rho(z) \geq d$ dan $\rho(w) \geq d$, yang mengakibatkan $z, w \in \rho_{d}$. Mengingat untuk setiap $a \in \rho(S), \rho_{a}$ adalah subsemiring dari $S$, maka diperoleh kondisi $z+w \in \rho_{d}$ dan $z w \in \rho_{d}$. Oleh karena itu,

$$
\rho(z+w) \geq d=a \wedge c=\rho(z) \wedge \rho(w) \text { dan } \rho(z w) \geq d=a \wedge c=\rho(z) \wedge \rho(w) .
$$

Dengan kata lain, $\rho$ adalah subsemiring fuzzy dari $S$.

Teorema 3.5 Misalkan $R$ adalah subsemiring dari semiring $S$, maka untuk setiap a $\in(0,1)$, terdapat subsemiring fuzzy $\rho$ dari $S$ sedemikian sehingga $\rho_{a}=R$.

Bukti:

Misalkan $\rho: S \rightarrow[0,1]$ adalah subsemiring fuzzy dari $S$ yang didefinisikan oleh

$$
\rho(x)= \begin{cases}a, & x \in R \\ 0, & x \in S \backslash R\end{cases}
$$

dengan $a \in(0,1)$. Oleh karena itu, berdasarkan fungsi keanggotaan $\rho$ diperoleh:

$$
\rho_{a}=\{x \in S \mid \rho(x) \geq a\}=\{x \in S \mid x \in R\}=R .
$$

Selanjutnya, andaikan ada $x_{0}, w_{0} \in S$ sedemikian sehingga dipenuhi kondisi

$$
\rho\left(x_{0}+w_{0}\right)<\rho\left(x_{0}\right) \wedge \rho\left(w_{0}\right) \text { dan } \rho\left(x_{0} w_{0}\right)<\rho\left(x_{0}\right) \wedge \rho\left(w_{0}\right) .
$$

Karena $|\rho(S)|=2$, maka

dan

$$
\rho\left(x_{0}+w_{0}\right)=0 \text { dan } \rho\left(x_{0}\right) \wedge \rho\left(w_{0}\right)=a
$$

Akibatnya,

$$
\rho\left(x_{0} w_{0}\right)=0 \text { dan } \rho\left(x_{0}\right) \wedge \rho\left(w_{0}\right)=a
$$

$$
x_{0}+w_{0} \notin R \text { dan } x_{0} w_{0} \notin R \text { tetapi } \rho\left(x_{0}\right)=\rho\left(w_{0}\right)=a \text {, yaitu } x_{0}, w_{0} \in R \text {. }
$$


Kondisi, $x_{0}+w_{0} \notin R$ dan $x_{0} w_{0} \notin R$ tetapi $x_{0}, w_{0} \in R$, kontradiksi dengan $\mathrm{R}$ adalah subsemiring dari $\mathrm{S}$, sehingga pengandaian salah, seharusnya untuk setiap $x, w \in S$ berlaku:

$$
\rho(x+y) \geq \rho(x) \wedge \rho(w) \text { dan } \rho(x y) \geq \rho(x) \wedge \rho(w) .
$$

Dengan kata lain, $\rho$ adalah subsemiring fuzzy dari $\mathrm{S}$.

Teorema 3.6 Misalkan $\rho$ adalah subsemiring fuzzy dari semiring $S$, maka

$$
\rho(z)=\sup \left\{a \in[0,1] \mid z \in \rho_{a}\right\}
$$

untuk setiap $z \in S$.

\section{Bukti:}

Misalkan $c \stackrel{\text { def }}{=} \sup \left\{a \in[0,1] \mid z \in \rho_{a}\right\}$, maka untuk sembarang $\epsilon>0, c-\epsilon<a$ untuk suatu $a \in[0,1]$ sedemikian sehingga $z \in \rho_{a}$ dan mengakibatkan $c-\epsilon<\rho(z)$. Karena $\epsilon$ adalah bilangan positif sembarang, maka dipenuhi kondisi $c \leq \rho(z)$. Selanjutnya, dimisalkan $\rho(z)=d$ maka

Oleh karena itu, $z \in \rho_{d}$ dan $d \in\left\{a \in[0,1] \mid z \in \rho_{a}\right\}$.

Berdasarkan hasil analisa di atas,

$$
\rho(z)=d \leq \sup \left\{a \in[0,1] \mid z \in \rho_{a}\right\}=c .
$$

$$
\rho(z)=\sup \left\{a \in[0,1] \mid z \in \rho_{a}\right\} .
$$

Berikut disajikan konvers dari Teorema 3.6. Misalkan $\Omega$ adalah subset tidak kosong dari $[0,1]$

Teorema 3.7 Misalkan $S$ adalah semiring dan $\left\{\delta_{a} \mid a \in \Omega\right\}$ adalah koleksi subsemiring dari $S$ sedemikian sehingga

1) $S=\bigcup_{a \in \Omega} \delta_{a}$

2) $a>c$, untuk setiap $a, c \in \Omega$ jika dan hanya jika $\delta_{a} \subset \delta_{c}$

Didefinisikan subset fuzzy $\rho$ dari $S$, untuk setiap $z \in S$,

$$
\rho(w) \stackrel{\text { def }}{=} \sup \left\{a \in \Omega \mid z \in \delta_{a}\right\}
$$

maka $\rho$ adalah subsemiring fuzzy dari $S$.

\section{Bukti:}

Untuk setiap $c \in[0,1]$, akan ditinjau dua kasus berikut

1) $c=\sup \{a \in \Omega \mid a<c\}$,

2) $c \neq \sup \{a \in \Omega \mid a<c\}$.

Untuk kasus (1), dipenuhi kondisi

$$
\begin{aligned}
z \in \rho_{c} & \Leftrightarrow z \in \rho_{a} \text { untuk setiap } a<c \\
& \Leftrightarrow z \in \bigcap_{a<c} \delta_{a},
\end{aligned}
$$

Oleh karena itu, $\rho_{c}=\bigcap_{a<c} \delta_{a}$ adalah subsemiring dari S.

Untuk kasus (2), terdapat $\epsilon>0$ sedemikian sehingga

$$
(c-\epsilon, c) \cap \Omega=\emptyset \text {. }
$$

Selanjutnya, diklaim $\rho_{c}=\bigcup_{a \geq c} \delta_{a}$. Diambil sembarang $z \in \bigcup_{a \geq c} \delta_{a}$, maka $z \in \delta_{a}$ untuk suatu $a \geq c$. Oleh karena itu, $\rho(z) \geq a \geq c$, yang mengakibatkan $z \in \rho_{c}$, yaitu $\bigcup_{a \geq c} \delta_{a} \subseteq \rho_{c}$. Sebaliknya, untuk $z \notin \bigcup_{a \geq c} \delta_{a}$, maka $z \notin \delta_{a}$ untuk setiap $a \geq c$, yang mengakibatkan $z \notin \delta_{a}$ untuk setiap $a>c-\epsilon$. Akibatnya, jika $z \in \delta_{a}$ maka $a \leq c-\epsilon$, sehingga dipenuhi kondisi $\rho(z) \leq c-\epsilon$ dan $z \notin \rho_{c}$. Oleh karena itu, $\rho_{c}=\bigcup_{a \geq c} \delta_{a}$ adalah subsemiring dari S.

Berdasarkan hasil Analisa di atas, diperoleh $\rho_{c}$ adalah subsemiring dari $S$, untuk setiap $c \in[0,1]$. Akibatnya, berdasarkan Teorema 3.4, diperoleh $\rho$ adalah subsemiring fuzzy dari $S$. 


\section{Kesimpulan}

Berdasarkan hasil dan pembahasan, diperoleh sifat penting yang menghubungkan antara subsemiring dan subsemiring fuzzy, yaitu subset fuzzy $\rho$ dari semiring $S$ adalah subsemiring fuzzy dari $S$ jika dan hanya level subset $\rho_{a}(\neq \varnothing)$ adalah subsemiring dari $S$, untuk setiap $a \in \rho(S)$.

\section{Referensi}

[1] A. Rosenfeld, 1971, Fuzzy groups, J. Math. Anal. Appl., vol. 35, no. 3, pp. 512517.

[2] L. A. Zadeh, 1965, Fuzzy Sets, Inf. Control, vol. 8, no. 3, pp. 338353.

[3] D. Mandal, 2014, Fuzzy Ideals and Fuzzy Interior Ideals in Ordered Semirings, Fuzzy Inf. Eng, vol. 6, pp. 101114.

[4] S. Abdurrahman, 2019, Image ( Pre-image) Homomorfisme Interior Subgrup Fuzzy, vol. 8, no. 1, pp. 1518.

[5] Y. B. Jun, M. Sapanci, and M. A. zt rk, 1998, Fuzzy ideals in gamma near-rings, Turkish J. Math., vol. 22, no. 4, pp. 449459.

[6] S. Abdurrahman, Interior Subgrup Fuzzy, J. Fourier, vol. 7, no. 1, pp. 1321, 2018.

[7] J. Ahsan, J. N. Mordeson, and M. Shabir, 2012, Fuzzy Semirings with Applications to Automata Theory. Springer Berlin Heidelberg New York Dordrecht London.

[8] R. D. Jagatap, 2014, Right k -Weakly Regular -Semirings, Hindawi Publ. Corp. Algebr., vol. 2014, pp. 1-5.

[9] J. Mordeson and K. R. Bhutani, 2005, Fuzzy Subsets and Fuzzy Subgroups, in Group, vol. 39, no. X, pp. 139. 
THIS PAGE INTENTIONALLY LEFT BLANK 